\title{
White blood cell differential fluorescence abnormal scattergram: A useful indicator for early detection of malarial parasite
}

\author{
Madeeha Rehan', Attika Khalid ${ }^{2}$, Fariha Nasreen ${ }^{3}$
}

\begin{abstract}
Background \& Objective: Undiagnosed malarial infectionis associated with significant mortality and morbidity. Laboratory investigations leading to rapid, accurate and timely diagnosis of malaria is still a challenge. This study was done to assess the utility of abnormal White blood cell differential fluorescence (WDF) scattergram for diagnosis of malaria. Our aim was to study the utility of WDF scattergram for early detection of malarial parasite.

Methods: All EDTA anti-coagulated blood samples received in laboratory during a period from Dec 2019 to May 2020 were analyzed on anautomated hematology analyzer, Sysmex XN 1000. All abnormal WDF scattergrams pertaining to plasmodium specie were critically evaluated and recorded. Review of Leishmanstained peripheral smears as well as immune-chromatographic assay by rapid test devices (RTD) was done. Accordingly, sensitivity, specificity, positive predictive value (PPV) and negative predictive value (NPV) for detection of malaria by abnormal scattergram were calculated.

Results: Out of total 1, 26,000of samples analyzed, abnormal WDF scattergrams were detected in 96 cases. Amongst these, 95.8\% (92) were positive for MP on Leishman-stained peripheral smear as well as on ICT with a p-value of 0.05 . WDF scattergram abnormalities typical of malaria showed a sensitivity of $80 \%$ and specificity of $93.26 \%$. Positive predictive value of $95.8 \%$ whereas negative predictive value of $99.9 \%$ was detected. Significant findings of hemolysis, platelet clumps, nucleated RBCS (NRBCs) and RBC agglutination were noted in cases $(n=4)$ with abnormal WDF scattergram negative for malaria on peripheral smear.

Conclusion: Interpretation of abnormal WDF scattergram not only increases the early detection rate for malarial parasite but isa strong indicator for presence of hemolysis, RBC agglutination, platelet clumps and leucoerythroblastic blood picture as well.
\end{abstract}

KEYWORDS: Malaria, Abnormal scattergram, Sysmex XN-1000.

doi: https://doi.org/10.12669/pjms.38.3.4702

How to cite this:

Rehan M, Khalid A, Nasreen F. White blood cell differential fluorescence abnormal scattergram: A useful indicator for early detection of malarial parasite. Pak J Med Sci. 2022;38(3):687-691. doi: https://doi.org/10.12669/pjms.38.3.4702

This is an Open Access article distributed under the terms of the Creative Commons Attribution License (http://creativecommons.org/licenses/by/3.0), which permits unrestricted use, distribution, and reproduction in any medium, provided the original work is properly cited.

1. Dr. Madeeha Rehan, Associate Professor,

2. Dr. Attika Khalid, Assistant Professor,

3. Dr. Fariha Nasreen, Postgraduate trainee Haematology,

1-3: Department of Pathology, Foundation University Medical College, Fauji Foundation Hospital Rawalpindi, Pakistan.

Correspondence:

Dr. Madeeha Rehan,

Adress: House\#310, Sector3, Street 13,

Airport Housing Society, Chaklala, Rawalpindi, Pakistan. Email: r.madeeha138@gmail.com

* Received for Publication:

May 11, 2021

* $1^{\text {st }}$ Revision Received:

* $2^{\text {nd }}$ Revision Received:

* Final Revision Accepted:
September 2, 2021

September 17, 2021

September 30, 2021

\section{INTRODUCTION}

Despite advances in diagnosis and management of malaria over the past decade, it remains the most common and important human vector-borne disease worldwide. Amongst the four known species of plasmodium $(\mathrm{P})$ globally, the dominant species is P. falciparum however P.vivax is the most commonly found species in Afghanistan, Pakistan, Iran and Iraq. In Pakistan, 1.5 million cases per annum have been estimated. In different provinces of Pakistan, the prevalence of P.vivax found to be $2.4 \%$ in Punjab, $10.8 \%$ in Sindh whereas prevalence of P.falciparum ranged from $0.1 \%$ in Islamabad and $3.8 \%$ in Balochistan. ${ }^{1}$ 
Microscopy of Giemsa-stained peripheral smears of patients with suspected malaria are still gold standard for its diagnosis. The liability of this method is based on the professional expertise in staining technique and microscopy. Moreover, it is also time consuming in setups with heavy workload and limited resources. Keeping these limitations in view, several new techniques for detection of malarial parasite were offered. These include antigen-coated dipstick tests, quantitative buffy coat examination using fluorescent dyes and polymerase chain reaction., Although these tests are sensitive and specific in malaria detection but are expensive and have less availability. ${ }^{4}$ Sensitivities of different immunologic methods for detection of malarial parasite is still a problem in detection of lower level of parasitaemia. ${ }^{5}$

Several latest automated haematology analyzers indicate flagging or graphical representation of malaria as a new emerging technique for its early detection. Automated haematology analyzers e.g., Sysmex XN-1000 perform a complete blood count and detect the presence of plasmodium species by showing an abnormality of the WDF, WNR, and RET scattergram. WDF scattergrams are graphical representation of the findings of the WDF channel. Different types of white blood cells are counted in WDF channel in XN series. ${ }^{6}$ It is based on principle of flowcytometry using a semiconductor laser to produce three types of optical data about different cells. Forward scatter light (FSL) as a cell size indicator, side scatter (SSC) as an indicator of the complexity of internal structure such as granules and nuclear content is indicated by side fluorescence light (SFL). Hemozoin, which is produced during hemoglobin catabolism, is phagocytized by neutrophils and monocytes leading to generation of abnormal scattergrams thus leading to the identification of malarial infection by automated methods.

Various studies have been conducted worldwide based on results of the automated hematology analyzers like, GEN S andLH750, XE2100 and XS100i7 for malarial detection. ${ }^{7}$ This study is an attempt to highlight the significance of WDF scattergram interpretation for early detection of malaria in unsuspected cases of malaria. It will not only be useful in underdeveloped countries, laboratories with increased workload and limited resources especially in endemic regions but also alarming for the pathologist to examine the peripheral blood smears more vigilantly thus preventing misdiagnosis and planning of timely therapeutic intervention for prevention of disease complications.

\section{METHODS}

It was a prospective study conducted at Fauji Foundation Hospital Rawalpindi over a period of six months duration from December 2019 to May 2020. After taking ethical approval from ethical review board (Ref. No. 410/ERC/FFH/Rwp, dated: 11/08/2020), all the complete blood counts (CBC) samples received in pathology laboratory from both outpatient departments (OPD) as well as wards were processed on automated Haematology analyzer Sysmex XN-1000. All the CBCs that were requested specifically for malaria and ICT MP were excluded from the studies. The CBCs showing abnormal scattergram pattern pertaining to malaria were selected and Leishman-stained peripheral smear stained were prepared and examined. ICT MP was also performed for all these selected cases. The abnormalities in the scattergram included double neutrophil line, multiple neutrophil lines, pseudo eosinophilia, right ward shift of ghost area and greying of the neutrophil/eosinophil zone. The peripheral smear was reviewed by post graduate resident and verified by consultant haematologist. All cases found to be positive for plasmodium species were documented in the software system and notified to the respective wards. The walk-in patients and patients from OPD were contacted on cell numbers to report to hospital immediately. Data analysis was done through SPSS version 17. All quantitative variables like age and gender were noted. Frequency and positive predictive and negative predictive value of malaria positive cases with abnormal scattergram was recorded. Sensitivity and specificity were also calculated for these selected cases.

\section{RESULTS}

A total of 1,26,000 CBCs received in hematology laboratory during the period of six months (Dec 2019-May 2020) were analyzed along with their respective WDF scattergrams. Of the total $C B C$, 75,600 received were of admitted cases in various wards, 44,100 cases were booked from outpatient department and6,300 CBC were booked directly in the laboratory as walk-in patients. Amongst the total cases, 96 CBC cases showed abnormal WDF scattergram pertaining to malaria. Of these 96 cases, 92 cases were positive for malarial parasite on peripheral smear and ICT with a p-value of 0.05 . $79.3 \%$ (73) were females and 20.6\% (19) were males with mean age of 40 years.

Amongst these, $93.7 \%$ (90) were found to be positive for MP on Leishman-stained peripheral 
Table I: Frequency of plasmodium specie observed in positive cases.

\begin{tabular}{llc}
\hline S.No. & Plasmodium specie & No. of Cases \\
\hline 1 & Plasmodium Vivax & 89 \\
2 & Plasmodium Falciparum & 01 \\
3 & Mixed infection with plasmo- & 02 \\
4 & dium Falciparum and Vivax & Nil \\
5 & Plasmodium Malariae & Nil \\
\hline 6 & Total & 92 \\
\hline
\end{tabular}

smear as well as on ICT MP. While 02 cases with no evidence of malaria on peripheral smear tested positive on ICT.

Out of the total 96 cases showing abnormal scatter gram, $4.1 \%$ (4) cases tested negative for malaria species on both peripheral smear as well as on ICT showed other findings such as RBC agglutination, platelet clumps, leucoerythroblastic blood picture and hemolysis on peripheral film examination (Table-II).

Amongst these cases only one case had plasmodium falciparum whereas mixed infection with plasmodium vivax and falciparum was detected in two cases. The rest of the 89 cases showed plasmodium vivax. No Ovale, Malariae species were reported (Table-I). Sensitivity, specificity, positive and negative predicted value were calculated (Table-III).

\section{DISCUSSION}

The detection of clinically unsuspected malaria is of significant importance in order to prevent the mortality and morbidity of the disease. ${ }^{8}$ There is usually limited access to clinical and laboratory expertise for identification, detection, and quantification of malaria in most endemic areas. Fluorescent and immunological tests are prohibitively expensive. ${ }^{4}$ In non-endemic countries, a differential diagnosis of malaria is usually not considered in acute febrile illness by most of the clinicians. ${ }^{5}$ In unsuspected patients, early detection
Table III: Positive and negative predictive value, sensitivity and specificity.

\begin{tabular}{lc}
\hline $\begin{array}{c}\text { Positive and negative predictive } \\
\text { value Sensitivity } \mathcal{E} \text { specificity }\end{array}$ & Frequency \\
\hline Positive predictive value & $95.8 \%$ \\
Negative predictive value & $96.8 \%$ \\
Sensitivity & $96.8 \%$ \\
Specificity & $99.9 \%$ \\
\hline
\end{tabular}

of malaria is an emerging screening tool by modern haematology analyzers.

In various studies Haematology analyzers evaluated for malaria detection include the CellDyn (Abbott Diagnostics, Santa Clara, CA), ${ }^{3}$ the GEN.S and LH-750 (Beckman Coulter, Miami, FL), ${ }^{4}$ and the XE-2100 and XS-1000i (Sysmex Corporation, Kobe, Kansai, Japan) ${ }^{6-8}$ Principle of flowcytometry is used in automated haematology analyzers and semiconductor laser is used to generate three types of optical data about cells. ${ }^{9}$ In this study a fundamental importance has been given to the malarial parasite detection by WDF abnormal scattergram (graphical representation of the findings of the WDF) generated by automated hematology analyzers XN-1000 for early detection of malaria even in absence of a specific clinical request. White cell differential channels in $\mathrm{XN}$ series is channel for counting the number of different white cell types present in the blood sample. In the DIFF channel (differential count) surfactant is used to lyse red blood cells and platelets and a polymethine dye to bind to nucleic acid which gives fluorescence signal intensity proportional to nucleic acid content. Furthermore, it uses an organic acid which specifically bind to eosinophil granules to be differentiated from neutrophils by high side scatter intensities. ${ }^{10,11}$

The present study showed abnormalities in hematological parameters like anemia, leukopenia and thrombocytopenia which were also reported in previous studies. ${ }^{12}$ Abnormal WDF scatter grams were detected in 96 cases. Out of these 96 cases, 90 were positive on peripheral smear as well as on ICT

Table-II: Findings of cases with abnormal scatter gram but negative ICT MP and peripheral smear.

\begin{tabular}{lcl}
\hline $\begin{array}{l}\text { Findings of cases with abnormal scatter gram but } \\
\text { negative ICT MP and peripheral smear }\end{array}$ & $\begin{array}{c}\text { Number of } \\
\text { cases (\%) }\end{array}$ & Observation \\
\hline Agglutination & 2 & Peripheral smear \\
Hemolysis & 1 & Gross examination and peripheral smear \\
Thrombocytosis and nucleated RBC & 1 & Peripheral smear \\
\hline
\end{tabular}


for MP. Two cases negative on peripheral smear were found to be positive on ICT.

A study conducted in India by Ningombam A et al showed $99.2 \%$ parasite detection by abnormal scattergram on similar haematology analyzer used in present study thus showing comparable results. ${ }^{13}$

In present study 89 cases were positive for plasmodium vivax, two for falciparum and one was mixed infection. It is in accordance with most of the studies reported plasmodium vivax as the most common infection globally. P.vivax is the most common plasmodium infections in Pakistan but $\mathrm{P}$. falciparum and mixed species infections are also prevalent. ${ }^{1}$ In the present study, the sensitivity was $96.8 \%$ and specificity was found to be $99.9 \%$. This is in accordance with a study conducted by Pilley et al., which suggested that there was correlation between microscopy and the abnormal scatter-grams generated by haematology analyzer and reported $100 \%$ sensitivity and specificity. However in contrast to our study the results were generated on $\mathrm{XN}-30 .{ }^{14}$ Another study by Sharma et al. suggested a sensitivity of $83.78 \%$ and specificity of $94.82 \% .^{2}$ In a study conducted in South Korea on XE 2100 analyzer showed comparable results to our study in which Yoo et al. reported sensitivity and specificity of $46.20 \%$ and $99.70 \%$ respectively. However, the main focus in their study has been only pseudo eosinophilia as a predictive marker for malaria detection whereas in our study we included all abnormal patterns observed in WDF channel. ${ }^{15}$ Hänscheid et al. concluded in their study that in comparison to microscopy, scatter flow cytometry using Cell-Dyn 3000 instrument detected significantly more patients with malaria. ${ }^{16}$

A study conducted by Zuluaga GC performed on XE-2100 indicated that malaria alarm produced by abnormal WDF scattergram can be helpful for early malaria microscopic diagnosis. ${ }^{17}$

According to the study conducted by Buoro et al. nine patients were positive to Plasmodium falciparum, one to Plasmodium ovale, and one to Plasmodium malaria and three to Plasmodium vivax. Significant abnormalities were observed in both white blood cell differential (WDF) and white cell nucleated (WNR) scatter grams (sensitivity 0.64 and specificity 1.0) in nine samples with parasites at gametocyte or schizonts stage, while characteristic scattergram abnormalities were not detected in the 5 samples containing only parasites at the trophozoites stage. The specific variations of some cell population data (CPD) could be recorded (sensitivity 1.00 and specificity 0.91 ) in these samples. ${ }^{18}$
In another study of the 80 patients who presented with fever and suspicion of malaria, 29 patients were positive for malaria and 10 cases were diagnosed incidentally by the findings on the cell counter and were confirmed by Giemsa-stained blood smears. The sensitivity and specificity of the abnormalities detected in the WBC-Diff channel in detecting malaria is $82 \%$ and $100 \%$ respectively. Using WBC-BASO channel abnormality for initial diagnosis the sensitivity and specificity is 50\% and $92.5 \%$ respectively. ${ }^{19}$ According to a study by Pillai KR et al., P.vivax was the most dominant species $(60.8 \%)$. Greying of the both neutrophil and eosinophil populations was seen in $75 \%$ of vivax $(p$-value $=0.019)$ and $23.2 \%$ of falciparum cases. ${ }^{20}$ In present study positive predictive value of $95.8 \%$ whereas negative predictive value of $99.9 \%$ was calculated. It reflected similarity with the results of study conducted by Kumar S \& Naik P which showed a sensitivity of $80 \%$ and specificity of $93.26 \%$ whereas positive predictive value was found to be of $40 \%$ and a negative predictive value of $98.81 \%{ }^{6}$ The cases having abnormal scatter grams but negative for malarial parasite on both smear and ICT found to have evidence of hemolysis, RBC agglutinates NRBCs and leucoerythroblastic blood picture on peripheral smears thus indicating other underlying clinical disorders. Platelet clumps were also identified in few of these cases. Although it is evident from literature that almost $10 \%$ non-malaria cases with different underlying clinical conditions e.g., thalassemia, leukemia, hemolytic diseases of newborn and septicemia showed various abnormalities in WDF scattergram mimicking malaria $^{3}$ but there is scarcity in literature in this context. However, these findings are of significant importance for diagnosis of the serious underlying conditions and should not be overlooked. In another study conducted by Campuzano-zuluaga et al similar findings were described using P.vivax infected samples, however in contrast to our study an increase in highly fluorescent lymphocyte-coded along with elevated reticulocyte/IRF ratio events in the DIFF scatterplot in a $P$. falciparum-infected patients were also detected. ${ }^{7}$ A study conducted in Pakistan concluded that in endemic areas with unavailability of expert microscopists automated haematology analyzers can be used as a useful adjunct for timely clinical diagnosis of malaria. ${ }^{21}$

Limitations of the study: It is a single center study. This study should be extended on multicenter to better enhance and validate the research findings. 
Furthermore, this idea can also be replicated for different $C B C$ analyzers to assess their utility in this regard as well.

\section{CONCLUSION}

Timely detection of malaria is life-saving. Knowledge about interpretation of abnormal WDF scattergram generated by automated haematology analyzers and its application can be utilized as an adjunctive diagnostic tool for early diagnosis of malaria especially in unsuspected cases where diagnosis can be overlooked. It has the potential to facilitate the diagnostic approaches, and significantly impact therapeutic monitoring and efficacy of anti-malarial and even clinical trials. It can also serve as the ideal blood donor screening tool for presence of malaria especially in malariaendemic regions.

Acknowledgment: Author thanks the technologists for their cooperation in data collection.

Disclosure of conflict of interest: None.

Financial support: None.

\section{REFERENCES}

1. Yasinzai MI, Kakarsulemankhel JK. Prevalence of human malaria infection in Pakistani areas bordering with Iran. J Pak Med Assoc. 2013;63:313-316.

2. Sharma S, Sethi N, Pujani M, Kushwaha S, Sharma S, Sethi N, et al. Abnormal WBC scattergram: A clue to the Abnormal WBC scattergram: A clue to the diagnosis of malaria. Haematology. 2013,18(2):101-105(2);101. doi: 10.79/1607845412y.0000000029

3. Vaughan JL, Alli N, Havyarimana S, Benade E. Assessment of International Consensus Group for Haematology smear review rules among patients with Plasmodium falciparum malaria in Johannesburg, South Africa. Afr J Labor Med. 2018;7(1):715. doi: 10.4102/ajlm.v7i1.715

4. Mai C, Hurtado C, State B, Glamorgan S, Sharma RS, Delhi N, et al. A rapid dipstick antigen capture assay for the diagnosis of falciparum malaria. Bull World Health Org. 1996;74(1):47-54.

5. Briggs C, Costa A Da, Dip N, Tech M, Freeman L, Dip N, et al. Development of an Automated Malaria Discriminant Factor Using VCS Technology. Am J Clin Pathol. 2006;126:691-698.

6. Kumar S, Naik P. Usefulness of automated hematology analyzer Sysmex XN 1000 in detection of Malaria. Indian J Pathol Oncol. 2016;3(4):658-661.

7. Campuzano-zuluaga G, Alvarez-sanchez G, Escobar-gallo GE, Valencia-zuluaga LM, Ríos-orrego AM, Pabón-vidal A, et al Design of Malaria Diagnostic Criteria for the Sysmex XE-2100 Hematology Analyzer. Am J TropHyg. 2010;82(5):402-411.

8. Mubeen KH, Devadoss CW, Rangan RA, Gitanjali M. Automated Hematology Analyzers in Diagnosis of Plasmodium vivax Malaria : an Adjunct to Conventional Microscopy. Mediterr J Hematol Infec Dis. 2014;6(1):e2014034.

9. Campuzano-Zuluaga G, Hanscheid T, Grobusch MP. Automated haematology analysis to diagnose malaria. Malar J. 2010;9:346. doi: 10.1186/1475-2875-9-346

10. Roccaforte V, Liuzzi G, Porreca WP, Russo RM, Zavaroni E, Angelis ML De, et al. A case of malaria diagnosed accidentally with Sysmex XN-9000 automated analyzer. J Lab Pecise Med. 2019;(6):3-5.
11. Mukry SN, Saud M, Sufaida G, Shaikh K. Laboratory Diagnosis of Malaria : Comparison of Manual and Automated Laboratory Diagnosis of Malaria. Canad J Infect Dis Med Microbiol. 2017;(3):5-7. doi: 10.1155/2017/9286392

12. Dumas C, Bienvenu A, Girard S, Picot S, Debize G, Durand B. Automated Plasmodium detection by the Sysmex XN hematology analyzer. J Clin Pathol. 2018;71:594-599.

13. Ningombam A, Sarkar A, Acharya S, Chopra A, Kumar K, Subramanian A. Application of Sysmex XN-Series Automated Haematology Analyser in the Rapid Detection of Malaria. Indian J Hematol Blood Transfus. 2020;36(3):512-518. doi: 10.1007/s12288-020-01276-x

14. Pillay E, Khodaiji S, Bezuidenhout BC, Litshie M, Coetzer TL. Evaluation of automated malaria diagnosis using the Sysmex XN - 30 analyser in a clinical setting. Malaria J 2019;18:1-14. doi: 10.1186/s12936-019-2655-8

15. Yoo J, Song J, Lee K, Sun Y, Kim Y, Park TS, et al. Short Report: Automated Detection of Malaria-Associated Pseudoeosinophilia and Abnormal WBC Scattergram by the Sysmex XE-2100 Hematology Analyzer: A Clinical Study with 1,801 Patients and Real-Time Quantitative PCR Analysis in Vivax MalariaEndemic Area. Am J Trop Med Hyg. 2010;82(3):412-414.

16. Hanscheid T, Langin M, Lell B, Potschke M, Oyakhirome S, Kremsner P. Full blood count and haemozoin-containing leukocytes in children with malaria: diagnostic value and association with disease severity. Malaria J. 2008;7:109. doi: 10.1186/1475-2875-7-109

17. Zuluaga GC, Hanscheid T, Grobusch MP. Automated haematology analysis to diagnose Malaria. Malar J. 2010;9:346.

18. Buoro S, Manenti B, Seghezzi M, Moioli V, Bagorria M, Callegaro A, et al. Abnormal scattergrams and cell population data generated by fully automated hematological analyzers: New tools for screening malaria infection? Int J Lab Hematol. 2018;40 (3):326-334. doi: 10.1111/ijlh.12790

19. Jain M, Gupta S, Jain J, Grover RK. Usefulness of automated cell counter in detection of malaria in a cancer set up--our experience. Indian J Pathol Microbiol. 2012;55(4):467-473. doi: 10.4103/0377-4929.107782

20. Pillai KR, Pady A, Ramesh M. Utility of automated haematology analyzer scattergram in diagnosis of malaria. National J Labor Med. 2020;9(1):6-10.

21. Mukry SN, Saud M, Sufaida G, Shaikh K, Naz A, Shamsi TS.Laboratory Diagnosis of Malaria: Comparison of Manual and Automated Diagnostic Tests. Can J Infect Dis Med Microbiol 2017;2017:9286392. doi: 10.1155/2017/9286392.

\section{Authors' Contribution:}

MR: Literature search, manuscript writing, data acquisition, manuscript drafting and editing and critical review for intellectual content and final approval of the version to be published. Corresponding author and accountable for accuracy and integrity of work.

AK: Concept and design of the study, data acquisition and statistical analysis, critical review. FN: Data collection, Data analysis \& Data interpretation, critical review. 